\title{
Prediction of Optimal Daily Step Count Achievement from Segmented School Physical Activity
}

\author{
Ryan D. Burns, ${ }^{1}$ Timothy A. Brusseau, ${ }^{1}$ and James C. Hannon ${ }^{2}$ \\ ${ }^{1}$ Department of Exercise and Sport Science, College of Health, University of Utah, 250 S. 1850 E., HPER North, RM 241, \\ Salt Lake City, UT 84112, USA \\ ${ }^{2}$ College of Physical Activity and Sport Sciences, West Virginia University, 375 Birch Street, P.O. Box 6116, \\ Morgantown, WV 26505, USA
}

Correspondence should be addressed to Ryan D. Burns; ryan.d.burns@utah.edu

Received 29 July 2015; Accepted 1 December 2015

Academic Editor: Julio Diaz

Copyright (C) 2015 Ryan D. Burns et al. This is an open access article distributed under the Creative Commons Attribution License, which permits unrestricted use, distribution, and reproduction in any medium, provided the original work is properly cited.

Optimizing physical activity in childhood is needed for prevention of disease and for healthy social and psychological development. There is limited research examining how segmented school physical activity patterns relate to a child achieving optimal physical activity levels. The purpose of this study was to examine the predictive relationship between step counts during specific school segments and achieving optimal school (6,000 steps/day) and daily (12,000 steps/day) step counts in children. Participants included 1,714 school-aged children (mean age $=9.7 \pm 1.0$ years) recruited across six elementary schools. Physical activity was monitored for one week using pedometers. Generalized linear mixed effects models were used to determine the adjusted odds ratios (ORs) of achieving both school and daily step count standards for every 1,000 steps taken during each school segment. The school segment that related in strongest way to a student achieving 6,000 steps during school hours was afternoon recess $(\mathrm{OR}=40.03 ; P<0.001)$ and for achieving 12,000 steps for the entire day was lunch recess $(\mathrm{OR}=5.03 ; P<0.001)$. School segments including lunch and afternoon recess play an important role for optimizing daily physical activity in children.

\section{Introduction}

Physical activity has numerous benefits in children including attenuation of chronic disease risk [1], improved motor development [2], improved classroom behavior [3], and improved cognitive functioning leading to better performance in the classroom [4]. Over the past few decades, numerous research studies have examined the correlates of physical activity in children [5]. The pedometer has been and continues to be a popular instrument for physical activity assessment. Recently it has been suggested that 12,000 steps should be a daily step count cut-point used to assess optimal physical activity levels in children [6]. Because children spend a significant proportion of weekdays in school settings, it is reasonable to expect that at least one-half of this daily recommendation should be met during school hours (i.e., 6,000 steps). Given the relative novelty of the aforementioned recommendations, there has been a paucity of research studies examining how various segments of the school day relate to a child achieving these standards.

Even though the majority of school hours are spent in sedentary behaviors, there are various segments throughout the school day where children can engage in physical activity. Research has shown that school segments such as PE, recess, and classroom activity breaks can provide opportunities for the child to engage in active play to increase their daily physical activity levels [7]. Before school and after school are other leisure times to engage in active play; however these segments are not typically considered "school hours" and are highly variable from day to day because of varying after school activities and transportation schedules. Of the school segments specifically included during school hours, recess has shown to have a significant impact on physical activity in children $[8,9]$. Various intervention studies have been employed over the last couple of decades and have shown increases in moderate-to-vigorous physical activity (MVPA) 
during recess, especially when increasing the number of unfixed equipment available on school grounds [10]. Most students have access to at least one recess per school day, but recess times have been cut in some school districts in the USA in favor for academic classes [11].

Classroom activity breaks can also increase physical activity in children [12]. Classroom breaks such as Energizers or implementation of programs such as TAKE 10! have shown to be significantly effective in increasing daily physical activity [13]. However, this specific school segment can also be highly variable from day to day because of classroom teacher's compliance with implementing these breaks in addition to competition with other school priorities $[14,15]$.

Physical education is a school subject vital for the physical, social, and psychological development of the child. Unfortunately, like recess, $\mathrm{PE}$ is being cut to prioritize more time in academic classes [16]. Indeed, most children are lucky to receive 40 minutes per week in PE and only a small proportion of schools in the USA implement daily PE curricula [17]. This significantly limits the amount of time a child can participate in active play to increase their daily physical activity during school hours; thus PE itself may have a small effect on improving the odds that a child achieves the 12,000-step physical activity standard.

Each of the aforementioned school segments has the potential to increase physical activity behaviors of schoolaged children. Given the lack of research examining how various school segments influence achievement of optimal school (6,000 steps) and daily (12,000 steps) physical activity levels, examining these relationships is a priority using large population samples of elementary school children. Identifying which school segments relate in strongest way to step count standards will provide information on how to more efficiently optimize physical activity in children during school hours and may provide evidence for future interventions to prioritize increasing physical activity behaviors during specific segments of the school day. Therefore, the purpose of this study was to examine the predictive relationship between school segment step counts and the odds of achieving school and daily step count standards in children. It was hypothesized that school segments such as lunch and afternoon recess will significantly relate to a child achieving the school and daily step count standards.

\section{Methods}

2.1. Participants. Participants were a convenience sample of 1,714 school-aged children recruited from six elementary schools located in a large metropolitan area in the Southwestern USA. The mean age of the sample was $9.7 \pm 1.0$ years and there were 926 girls and 788 boys who participated. The racial composition of the sample was $43 \%$ Caucasian, $44 \%$ Hispanic, 7\% African American, 4\% American Indian, and $2 \%$ Asian or Pacific Islander. Written assent was obtained from the students and written consent was obtained from the parents prior to data collection. The University Institutional Review Board approved the protocols used in this study.
2.2. Instrumentation. The Yamax SW-200 pedometer was used to measure children's physical activity. This model has produced valid and reliable scores in measuring children's physical activity in field settings [18]. Prior to data collection, the pedometers were checked for test-retest reliability using a series of shake tests, developed by Vincent and Sidman [19]. Additionally, all participants completed a walking test [20] to ensure that the pedometers accurately measured steps. Classroom and PE teachers provided opportunities for students to practice wearing the devices before data collection to attenuate risk for behavioral reactivity. On the first day of data collection, a five-minute review of the pedometer protocol was given by a member of the research team addressing how to (a) place pedometers on the body, (b) remove the pedometers before engaging in water activities and sleeping, and (c) reattach the pedometer each morning upon dressing for the school day. Participants were instructed to wear their pedometers at all times before, during, and after school hours while participating in their normal daily activities except during water activities and sleeping.

2.3. Procedures. Parents completed a background questionnaire used to collect demographic information including the child's age, sex, and ethnicity. Height (to the nearest $0.5 \mathrm{~cm}$ ) and weight (to the nearest $0.1 \mathrm{~kg}$ ) were measured using a digital scale (Seca 882 Digital BMI Scale, Hanover, MD, USA) and stadiometer (Seca 214, Hanover, MD, USA). At the beginning of the school day, students were prompted to record their pedometer step counts from the previous day and reset their pedometer for the current day. At the beginning and end of each school segment and at the end of each school day students were also prompted to record their step counts. During data collection, research team members actively provided prompts reminding the students how to correctly wear the instrument. Research team members scanned student responses on their recorded step count data and questioned children if step count outliers and extreme scores were detected. After given the information regarding recorded step counts from the previous day, the researchers entered the data into an Excel spreadsheet. If pedometers were lost, broken, or misused, students were prevented from getting another pedometer ( $<5 \%$ of participants). Furthermore, students participated in random daily validation checks by completing a brief survey about their previous day's physical activity. Survey question items required a yes or no response regarding if they wore their pedometer the entire day and if they removed the pedometer for any reason other than showering, swimming, or sleeping. The pedometer data collection process was repeated daily across five days of the school week at each of the six schools.

2.4. Statistical Analysis. Data were screened for outliers using $z$-scores and boxplots and checked for Gaussian distributions using histograms and $k$-density plots. Differences between the sexes and grade levels on step counts within each school segment were examined using $2 \times 2$ Factorial ANOVA tests. Alpha level was adjusted using the Bonferroni method to account for analysis on multiple dependent variables. Effect 
TABLE 1: Descriptive statistics for the total sample and within sex and grade groups (means and standard deviations).

\begin{tabular}{|c|c|c|c|c|c|}
\hline & $\begin{array}{c}\text { Total sample } \\
(N=1,714)\end{array}$ & $\begin{array}{c}\text { Girls } \\
(n=926)\end{array}$ & $\begin{array}{c}\text { Boys } \\
(n=788)\end{array}$ & $\begin{array}{l}\text { Grade } 4 \\
(n=816)\end{array}$ & $\begin{array}{l}\text { Grade } 5 \\
(n=898)\end{array}$ \\
\hline $\mathrm{BMI}^{\mathrm{a}}\left(\mathrm{kg} / \mathrm{m}^{2}\right)$ & $19.6(4.2)$ & $19.1(4.1)$ & $19.6(4.3)$ & $19.1(4.3)$ & $19.6(4.0)$ \\
\hline $\mathrm{PE}^{\mathrm{b}}$ steps & $1,897(808)$ & $1,823(781)$ & $1,979^{\circ}(831)$ & $1,890(771)$ & $1,953(873)$ \\
\hline Lunch recess steps & $1,537(523)$ & $1,374(456)$ & $1,706^{\circ}(536)$ & $1,512(484)$ & $1,614^{\bullet}(534)$ \\
\hline Afternoon recess steps & $1,281(541)$ & $1,184(504)$ & $1,394^{\circ}(561)$ & $1,313(499)$ & $1,266(560)$ \\
\hline Classroom steps & $2,052(1,566)$ & $1,850(1,390)$ & $2,261(1,707)$ & $1,931(1,540)$ & $2,037(1,560)$ \\
\hline School steps & $5,625(1,855)$ & $5,242(1,712)$ & $6,018^{\circ}(1,915)$ & $5,139(1,687)$ & $6,106^{\circ}(1,865)$ \\
\hline Total day steps & $13,048(4,248)$ & $10,218(3,195)$ & $12,088^{\circ}(3,895)$ & $12,403(4,306)$ & $13,578^{\circ}(4,071)$ \\
\hline
\end{tabular}

Note. ${ }^{a}$ BMI stands for Body Mass Index; ${ }^{b}$ PE stands for physical education; bold denotes statistical significance between sexes or between grade levels, ${ }^{\circ} P<$ 0.001 .

sizes were also calculated for each pair-wise comparison using Cohen's $d$. Effect sizes were considered small if $d<0.20$, medium if $d \approx 0.50$, and large if $d \geq 0.8$ [21].

The primary analysis consisted of employing generalized linear mixed effects models with a logit link function to examine the odds of achieving school and daily step count standards per unit change in a school segment step count. The daily standard was 12,000 steps per day as recommended by Colley et al. [6], and the school standard was 6,000 steps per day. School segment step count data was converted from absolute steps to thousands of steps in order to interpret the predictor variables in meaningful units. The student unit of measurement was clustered within individual classrooms; therefore classrooms were a higher level and treated as a random effect. Although there were multiple schools in the data set, it was not treated as a higher level with an additional random effect because of the small number clusters. This decision was supported by a nonsignificant likelihood ratio chi-square statistic that tested if the model including a school-level and teacher-level random effect was statistically different compared to a model with a teacher-level random effect only. Additional variables that were entered into each model included sex, grade, and ethnicity to control for any modifying effects. The reported results included an adjusted odds ratio for meeting the school day or total day step count standard per every one thousand step increase within a specific school segment. All analyses had an initial alpha level set at $P \leq 0.05$ and were carried out using STATA v14.0 statistical software package (College Station, TX, USA).

\section{Results}

The descriptive statistics for the total sample and within each sex and grade level are presented in Table 1. Boys took statistically more steps than girls during PE (mean difference $=$ 156 steps, $P<0.001$, Cohen's $d=0.18$ ), lunch recess (mean difference $=332$ steps, $P<0.001$, Cohen's $d=0.62$ ), and afternoon recess (mean difference $=210$ steps, $P<0.001$, Cohen's $d=0.37$ ) and took more steps during the school day (mean difference $=776$ steps, $P<0.001$, Cohen's $d=0.40$ ) and during the entire day (mean difference $=1,870$ steps, $P<$ 0.001 , Cohen's $d=0.48$ ). Children in the fifth grade took more steps than children in the fourth grade during lunch recess (mean difference $=102$ steps, $P<0.001$, Cohen's $d=0.19$ )
TABle 2: Parameter estimates from the final fixed effects model solution using the school step target criterion (6,000 steps).

\begin{tabular}{lccc}
\hline & $\begin{array}{c}\text { Adjusted } \\
\text { odds ratio }\end{array}$ & $95 \% \mathrm{CI}^{\mathrm{b}}$ & $P$ value \\
\hline PE $^{\text {a }}$ steps & $\mathbf{3 . 3 9}^{\circ}$ & $(1.82,6.29)$ & $<0.001$ \\
Lunch recess steps & $\mathbf{2 6 . 9 0}^{\circ}$ & $(6.03,119.89)$ & $<0.001$ \\
Afternoon recess steps & $\mathbf{4 0 . 0 3}^{\circ}$ & $(9.90,161.75)$ & $<0.001$ \\
Classroom steps & $\mathbf{1 4 . 2 1}^{\circ}$ & $(6.43,31.40)$ & $<0.001$ \\
Female & 0.72 & $(0.29,1.78)$ & 0.475 \\
4th grade & 1.15 & $(0.42,3.22)$ & 0.779 \\
Hispanic & 0.21 & $(0.04,1.19)$ & 0.080 \\
African American & 1.54 & $(0.17,14.41)$ & 0.701 \\
American Indian & 0.57 & $(0.05,2.61)$ & 0.322 \\
Asian/Pacific Islander & 0.38 & $(0.05,2.61)$ & 0.322 \\
\hline
\end{tabular}

Note. ${ }^{\mathrm{a}} \mathrm{PE}$ stands for physical education; ${ }^{\mathrm{b}} 95 \% \mathrm{CI}$ stands for the $95 \%$ Confidence Interval; bold denotes statistical significance, ${ }^{\circ} P<0.001$.

and took more steps during the school day (mean difference $=$ 967 steps, $P<0.001$, Cohen's $d=0.50$ ) and during the entire day (mean difference $=1,175$ steps, $P<0.001$, Cohen's $d=$ 0.27 ). ANOVA tests revealed no statistically significant $\operatorname{sex} \times$ grade interactions.

The results from the generalized mixed effects models are presented in Tables 2 and 3 for school and daily standards, respectively. The odds of a child achieving school standards were 3.39 times higher if 1,000 more steps were taken during PE, 26.90 times higher during lunch, 40.03 times higher during recess, and 14.21 times higher during classroom activity breaks after controlling for sex, grade, race, and teacher-level clustering $(P<0.001)$. Regarding daily step count standards, the odds of achieving the daily standard were 5.03 times greater for every 1,000 steps taken during lunch, 3.70 times greater during recess, and 1.98 times greater during classroom activity breaks after controlling for sex, grade, race, and teacher-level clustering. Increases in step counts during PE did not yield any significant change in odds in achieving the daily standard during PE days. Figures 1 and 2 display a scatterplot with line of best fit showing the strongest school segment associations between afternoon recess steps and school steps and between lunch recess steps and daily steps, respectively. 
TABLE 3: Parameter estimates from the final fixed effects model solution using the daily step target criterion (12,000 steps).

\begin{tabular}{lccc}
\hline & $\begin{array}{c}\text { Adjusted } \\
\text { odds ratio }\end{array}$ & $95 \% \mathrm{CI}^{\mathrm{b}}$ & $P$ value \\
\hline PE $^{\mathrm{a}}$ steps & 1.03 & $(0.72,1.48)$ & 0.835 \\
Lunch recess steps & $\mathbf{5 . 0 3}^{\dagger}$ & $(1.85,13.74)$ & 0.002 \\
Afternoon recess steps & $\mathbf{3 . 7 0}^{\dagger}$ & $(1.53,8.78)$ & 0.003 \\
Classroom steps & $\mathbf{1 . 9 8}^{\dagger}$ & $(1.41,2.76)$ & $<0.001$ \\
Female & 0.82 & $(0.42,1.61)$ & 0.495 \\
4th grade & 0.76 & $(0.35,1.67)$ & 0.495 \\
Hispanic & 0.40 & $(0.12,1.29)$ & 0.126 \\
African American & 0.92 & $(0.19,4.37)$ & 0.921 \\
American Indian & 1.88 & $(0.90,3.93)$ & 0.090 \\
Asian/Pacific Islander & 3.38 & $(0.75,15.19)$ & 0.112 \\
\hline
\end{tabular}

Note. ${ }^{\mathrm{a}} \mathrm{PE}$ stands for physical education; ${ }^{\mathrm{b}} 95 \%$ CI stands for the $95 \%$ Confidence Interval; bold denotes statistical significance, ${ }^{\dagger} P<0.01$.

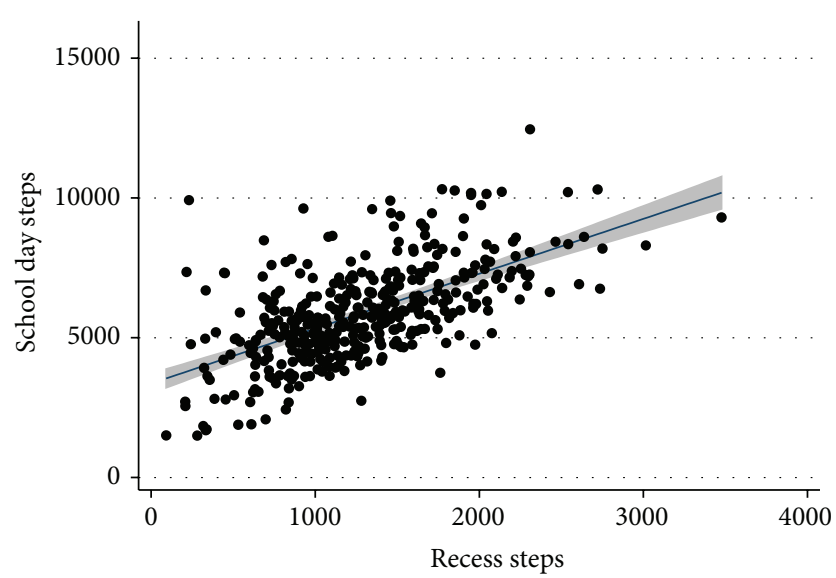

FIgURE 1: Scatterplot and line of best fit showing the linear relationship between afternoon recess steps and school steps $(r=0.61$; $P<0.001)$.

\section{Discussion}

The purpose of this study was to examine the predictive relationship between step counts taken during specific school segments and achievement of optimal school and daily physical activity levels. The results indicated that lunch and afternoon recess had the strongest predictive ability for determining the odds of achieving school and daily step count standards. Although all school segments related to achieving 6,000 steps during school and all but PE related to achieving 12,000 steps during the entire day, researchers and practitioners aiming to improve the odds that a child can achieve optimal levels of physical activity may want to prioritize school recess times because of the strong associations found in this study.

The primary finding from the current study was that the recess school segments had the strongest predictive relationship with a child achieving school and daily pedometer step count standards. Recess is considered a school leisure time where girls and boys have opportunities to participate

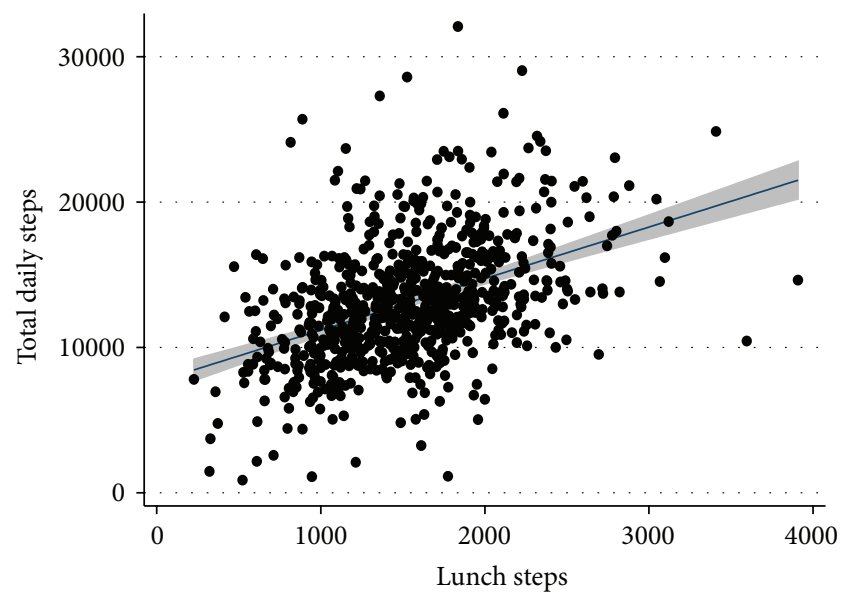

FIgURE 2: Scatterplot and line of best fit showing the linear relationship between lunch recess steps and total daily steps $(r=$ $0.44 ; P<0.001)$.

in active play. Structured and semistructured recess times have both been shown to increase physical activity levels in children. Increases in physical activity during recess are most significant when students are given unfixed equipment and are outdoors and upon providing students options to play in their preferred modes of physical activity [22]. It has been shown that urban school-aged girls take on average 976 steps and boys take approximately 1,281 steps during outdoor recess [23]. Brusseau and Kulinna [24] showed that fifth graders achieved the most steps during the entire day on the days they had multiple recess times and the least amount of steps on the days when they had just one recess. Indeed, recess has been shown to account for anywhere between $11 \%$ and $21 \%$ of their daily step counts and can account for as much as $40 \%$ of daily MVPA recommendations [25]. The results from the current study suggest that increasing physical activity by 1,000 steps during this school segment (recess) has the strongest predictive ability that a student will achieve at least 6,000 steps per school day and at least 12,000 steps during the entire day. Possible explanations why recess yielded the greatest odds of achieving physical activity guidelines include the frequency of physical activity exposure, as recess was daily and at multiple times at each school, the amount of play space and available play equipment for the children to use, and the number of other students/peers to interact with, as each recess time period typically involved several classes and grade levels. There may be discordance in the exposure of these factors compared to other school segments including $\mathrm{PE}$ or during classroom activity breaks where the frequency of physical activity exposure is more limited (e.g., PE is only once per week), the amount of play space tends to limited to gymnasium or classroom space, certain play equipment may be unavailable to use, and the number of other students to interact with is limited to peers within a child's respective classroom.

Other school leisure times that associated with the odds of achieving school and daily step count standards included classroom activity breaks. Classroom activity breaks have been shown to have a positive effect not only on physical 
activity but also on classroom behaviors and improving academic performance $[26,27]$. Unfortunately, studies have shown that individual teacher compliance with implementing activity breaks varies widely not only among different schools but also within the same school $[14,15]$. However, the results from this study clearly show that, even after controlling for demographic information and the effect of other school segments, classroom activity breaks still have a significant relationship with predicting if a student achieved step count standards. The results indicated that additional 1,000 steps from classroom activity breaks associate with 14 times higher odds that a child achieved 6,000 steps during the school day and almost doubled the odds that a child achieved 12,000 steps during the entire day. Despite these very strong associations, previous research interventions employing classroom activity breaks increase step counts, on average, between approximately 600 and 800 steps $[12,13]$. This study clearly showed that children took a significantly greater number of steps during classroom breaks (approximately 2,000 steps) compared to past research. This may be because most of the teachers in the current study implemented more than one 10minute break throughout the school day, as opposed to only one activity break per school day implemented in previous intervention work. Teachers in this study were recommended to employ at least two classroom activity breaks per day (one in the morning and one in the afternoon). Therefore, given the results from this and past studies, using classroom activity breaks to increase a child's step count by 1,000 steps, although certainly feasible, may not be the norm within a day-to-day context if only implementing one activity break per school day.

Although steps taken during PE did associate with students achieving school step count standards, it did not associate with achievement of daily standards after accounting for demographic information and the effect of the other school segments. In this study, PE was given once per week for 40 minutes. The number of steps taken during PE was approximately 2,000 steps for both sexes, which is more than what has been previously reported using population samples of similar age where steps ranging from approximately 1,200 to 1,400 steps were taken [28]. In the current study, the number of steps taken during PE was similar to those taken during classroom breaks and more than those taken during both recess times; however its relationship with achievement of daily levels was not statistically significant. One possible explanation for this was that there might have been a compensatory effect where children became inactive during other school segments as well as after school during the days that they had PE. It has been suggested that on restricted physical activity days (i.e., days without PE or recess times) there may be increased physical activity during other school segments and leisure times to achieve a balanced energy homeostasis [29]. It is therefore plausible that the opposite is true, where increased activity in PE may lead to decreased energy expenditure (i.e., decreased step counts) during recess or after school hours to maintain this energy homeostasis. However, the authors do not know of any other studies examining this phenomenon and therefore may be a priority for future research.
There are limitations to this study that must be considered before generalizations can be made. Because of the crosssectional nature of the current study, no casual links can be established, only correlational and predictive relationships. Also, only fourth and fifth grade students were examined; therefore the results cannot be generalized to younger or older age groups. This study used PE curriculum that was administered just one day per week at each of the six schools; therefore the results do not generalize to schools offering daily PE curricula. Finally, physical activity was monitored using pedometers; therefore physical activity of specific intensities was not examined in addition to physical activity modes nonambulatory in nature such as swimming, cycling, and resistance training.

In conclusion, school segments including lunch and afternoon recess most strongly relate to the odds that a child achieves 6,000 steps during school hours and achieves 12,000 steps during the entire day. Other school segments that relate to achieving the school step count standard include PE and classroom activity breaks and those relating to achieving the daily step count standard include classroom activity breaks. The results indicate that increasing school physical activity during most of the examined school segments has the potential to significantly increase the odds that a child can achieve optimal school and daily levels; however the recess times tended to have the strongest effect. Interventions aiming to increase school and daily physical activity levels in children may want to prioritize increasing these behaviors during recess because of the strong relationships seen in the current study. Indeed, optimizing physical activity levels in children is important for optimal physical and psychological development, and this study provides unique insights into how varying levels of physical activity during specific school segments relate to achieving current step count standards to improve their overall health and well-being.

\section{Conflict of Interests}

The authors declare that there is no conflict of interests regarding the publication of this paper.

\section{Acknowledgments}

The authors would like to thank the teachers and students who participated in this study.

\section{References}

[1] L. M. Boddy, M. H. Murphy, C. Cunningham et al., "Physical activity, cardiorespiratory fitness, and clustered cardiometabolic risk in 10- to 12-year-old school children: the REACH Y6 study," American Journal of Human Biology, vol. 26, no. 4, pp. 446-451, 2014.

[2] D. F. Stodden, Z. Gao, J. D. Goodway, and S. J. Langendorfer, "Dynamic relationships between motor skill competence and health-related fitness in youth," Pediatric Exercise Science, vol. 26, no. 3, pp. 231-241, 2014.

[3] M. T. Mahar, "Impact of short bouts of physical activity on attention-to-task in elementary school children," Preventive Medicine, vol. 52, supplement 1, pp. S60-S64, 2011. 
[4] D. M. Castelli, C. H. Hillman, S. M. Buck, and H. E. Erwin, "Physical fitness and academic achievement in third- and fifthgrade students," Journal of Sport \& Exercise Psychology, vol. 29, no. 2, pp. 239-252, 2007.

[5] J. F. Sallis, J. J. Prochaska, and W. C. Taylor, "A review of correlates of physical activity of children and adolescents," Medicine and Science in Sports and Exercise, vol. 32, no. 5, pp. 963-975, 2000.

[6] R. C. Colley, I. Janssen, and M. S. Tremblay, "Daily step target to measure adherence to physical activity guidelines in children," Medicine \& Science in Sports \& Exercise, vol. 44, no. 5, pp. 977982, 2012.

[7] T. L. McKenzie, N. C. Crespo, B. Baquero, and J. P. Elder, "Leisure-time physical activity in elementary schools: analysis of contextual conditions," Journal of School Health, vol. 80, no. 10, pp. 470-477, 2010.

[8] A. Beighle, C. F. Morgan, G. Le Masurier, and R. P. Pangrazi, "Children's physical activity during recess and outside of school," Journal of School Health, vol. 76, no. 10, pp. 516-520, 2006.

[9] J. Mota, P. Silva, M. P. Santos, J. C. Ribeiro, J. Oliveira, and J. A. Duarte, "Physical activity and school recess time: differences between the sexes and the relationship between children's playground physical activity and habitual physical activity," Journal of Sports Sciences, vol. 23, no. 3, pp. 269-275, 2005.

[10] N. D. Ridgers, J. Salmon, A.-M. Parrish, R. M. Stanley, and A. D. Okely, "Physical activity during school recess: a systematic review," American Journal of Preventive Medicine, vol. 43, no. 3, pp. 320-328, 2012.

[11] Center on Educational Policy, Instructional Time in Elementary Schools: A Closer Look at Changes for Specific Subjects, Center on Educational Policy, Washington, DC, USA, 2008.

[12] M. T. Mahar, S. K. Murphy, D. A. Rowe, J. Golden, A. T. Shields, and T. D. Raedeke, "Effects of a classroom-based program on physical activity and on-task behavior," Medicine and Science in Sports and Exercise, vol. 38, no. 12, pp. 2086-2094, 2006.

[13] T. L. Goh, J. Hannon, C. A. Webster, L. W. Podlog, T. Brusseau, and M. Newton, "Effects of a classroom-based physical activity program on children's physical activity levels," Journal of Teaching in Physical Education, vol. 33, no. 4, pp. 558-572, 2014.

[14] J. E. Donnelly, J. L. Greene, C. A. Gibson et al., "Physical Activity Across the Curriculum (PAAC): a randomized controlled trial to promote physical activity and diminish overweight and obesity in elementary school children," Preventive Medicine, vol. 49, no. 4, pp. 336-341, 2009.

[15] M. W. Martin, S. Martin, and P. Rosengard, "PE2GO: program evaluation of a physical activity program in elementary schools," Journal of Physical Activity and Health, vol. 7, no. 5, pp. 677-684, 2010.

[16] Centers for Disease Control and Prevention, The Association Between School-Based Physical Activity, Including Physical Education, and Academic Performance, US Department of Health and Human Services, Atlanta, Ga, USA, 2010.

[17] S. M. Lee, C. R. Burgeson, J. E. Fulton, and C. G. Spain, "Physical education and physical activity: results from the school health policies and programs study 2006," Journal of School Health, vol. 77, no. 8, pp. 435-463, 2007.

[18] J. P. Barfield, D. A. Rowe, and T. J. Michael, "Interinstrument consistency of the Yamax Digi-Walker pedometer in elementary school-aged children," Measurement in Physical Education and Exercise Science, vol. 8, no. 2, pp. 109-116, 2004.
[19] S. D. Vincent and C. L. Sidman, "Determining measurement error in digital pedometers," Measurement in Physical Education and Exercise Science, vol. 7, no. 1, pp. 19-24, 2003.

[20] C. Tudor-Locke, "Taking steps toward increase physical activity, using pedometers to measure and motivate," Research Digest, vol. 3, no. 7, pp. 1-8, 2002.

[21] J. Cohen, Statistical Power Analysis for the Behavioral Sciences, Lawrence Erlbaum Associates, Hillsdale, NJ, USA, 1998.

[22] J. N. Larson, T. A. Brusseau, B. Chase, A. Heinemann, and J. C. Hannon, "Youth physical activity and enjoyment during semistructured versus unstructured school recess," Open Journal of Preventive Medicine, vol. 4, no. 8, pp. 631-639, 2014.

[23] I. Tran, B. R. Clark, and S. B. Racette, "Physical activity during recess outdoors and indoors among urban public school students, St. Louis, Missouri, 2010-2011," Preventing Chronic Disease, vol. 10, Article ID 130135, 2013.

[24] T. A. Brusseau and P. H. Kulinna, "An examination of four traditional physical activity models on children's step counts and MVPA," Research Quarterly for Exercise and Sport, vol. 86, pp. 88-93, 2015.

[25] N. D. Ridgers, G. Stratton, and S. J. Fairclough, "Physical activity levels of children during school playtime," Sports Medicine, vol. 36, no. 4, pp. 359-371, 2006.

[26] J. R. Best, "Effects of physical activity on children's executive function: contributions of experimental research on aerobic exercise," Developmental Review, vol. 30, no. 4, pp. 331-351, 2010.

[27] D. McNaughten and C. Gabbard, "Physical exertion and immediate mental performance of sixth-grade children," Perceptual and Motor Skills, vol. 77, part 2, no. 3, pp. 1155-1159, 1993.

[28] B. L. Alderman, T. Benham-Deal, A. Beighle, H. E. Erwin, and R. L. Olson, "Physical education's contribution to daily physical activity among middle school youth," Pediatric Exercise Science, vol. 24, no. 4, pp. 634-648, 2012.

[29] D. Dale, C. B. Corbin, and K. S. Dale, "Restricting opportunities to be active during school time: Do children compensate by increasing physical activity levels after school?" Research Quarterly for Exercise and Sport, vol. 71, no. 3, pp. 240-248, 2000. 


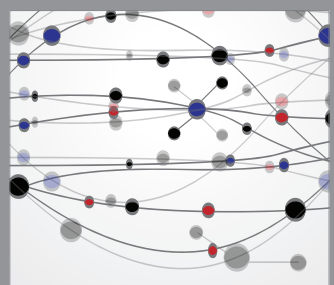

The Scientific World Journal
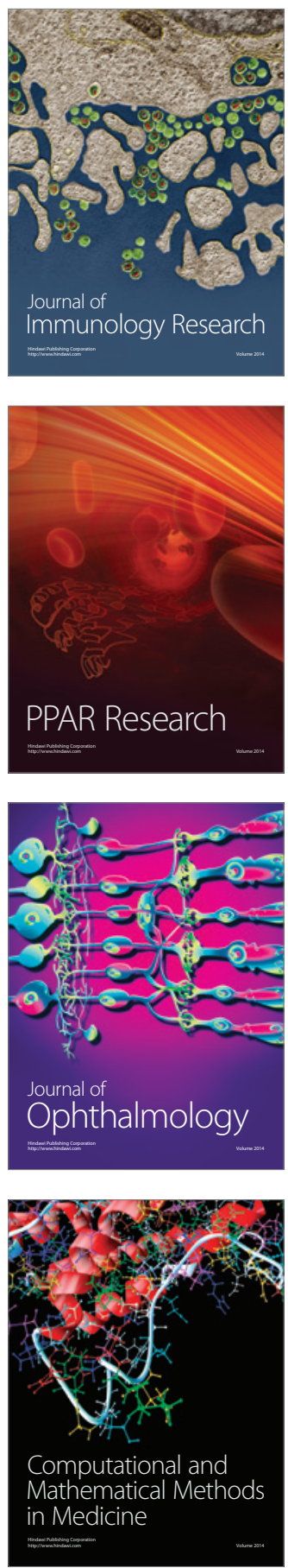

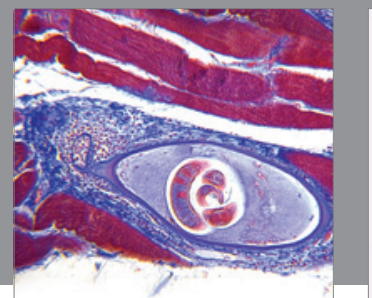

Gastroenterology

Research and Practice
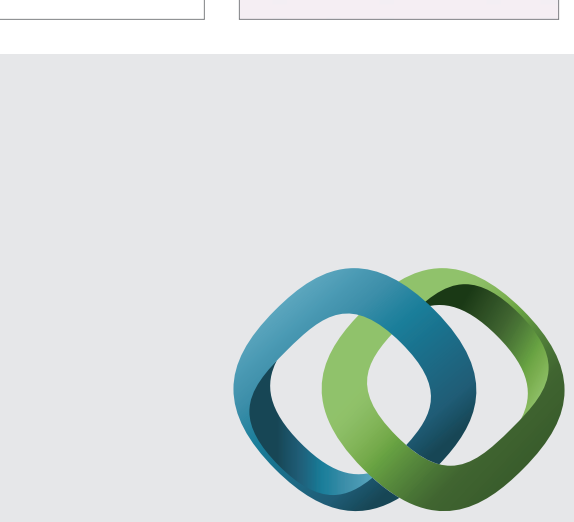

\section{Hindawi}

Submit your manuscripts at

http://www.hindawi.com
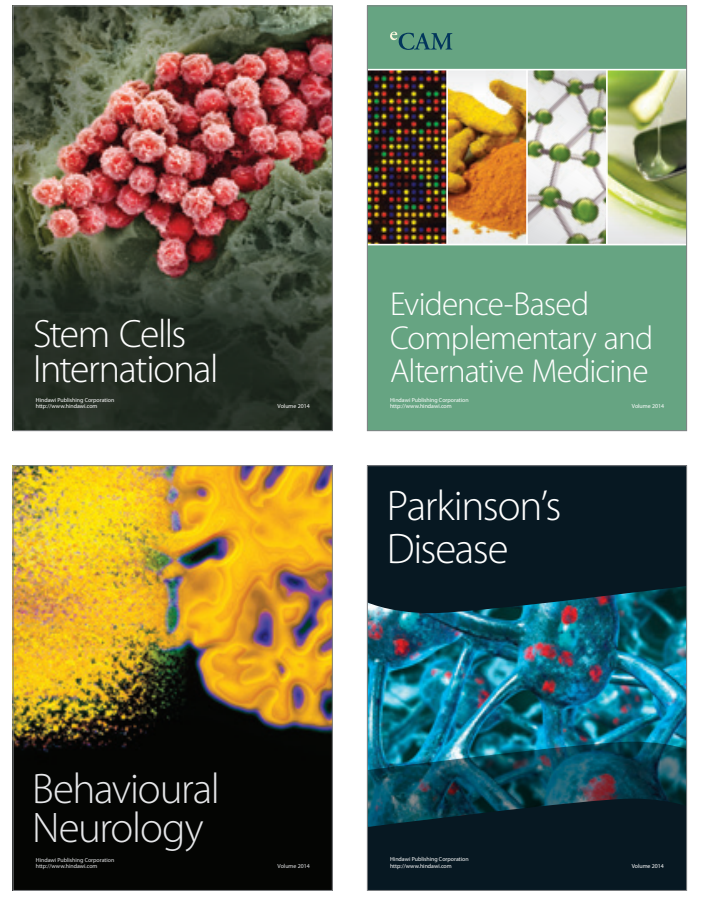
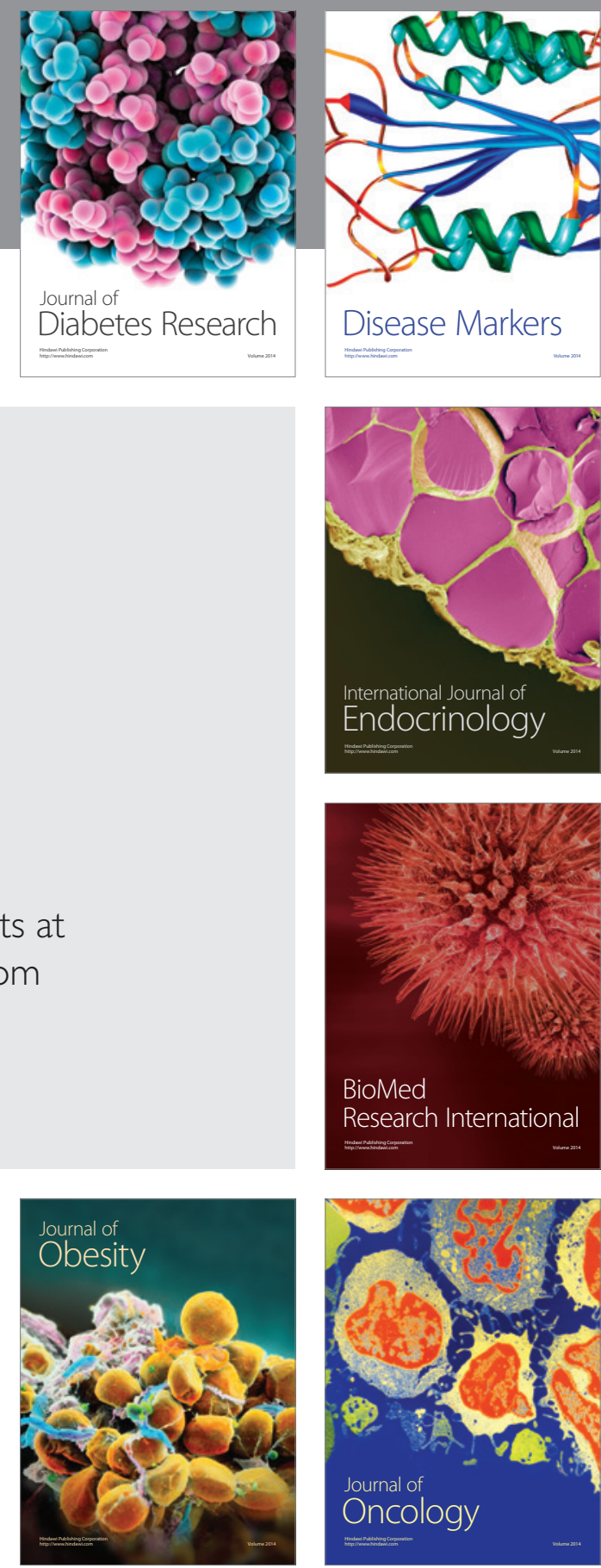

Disease Markers
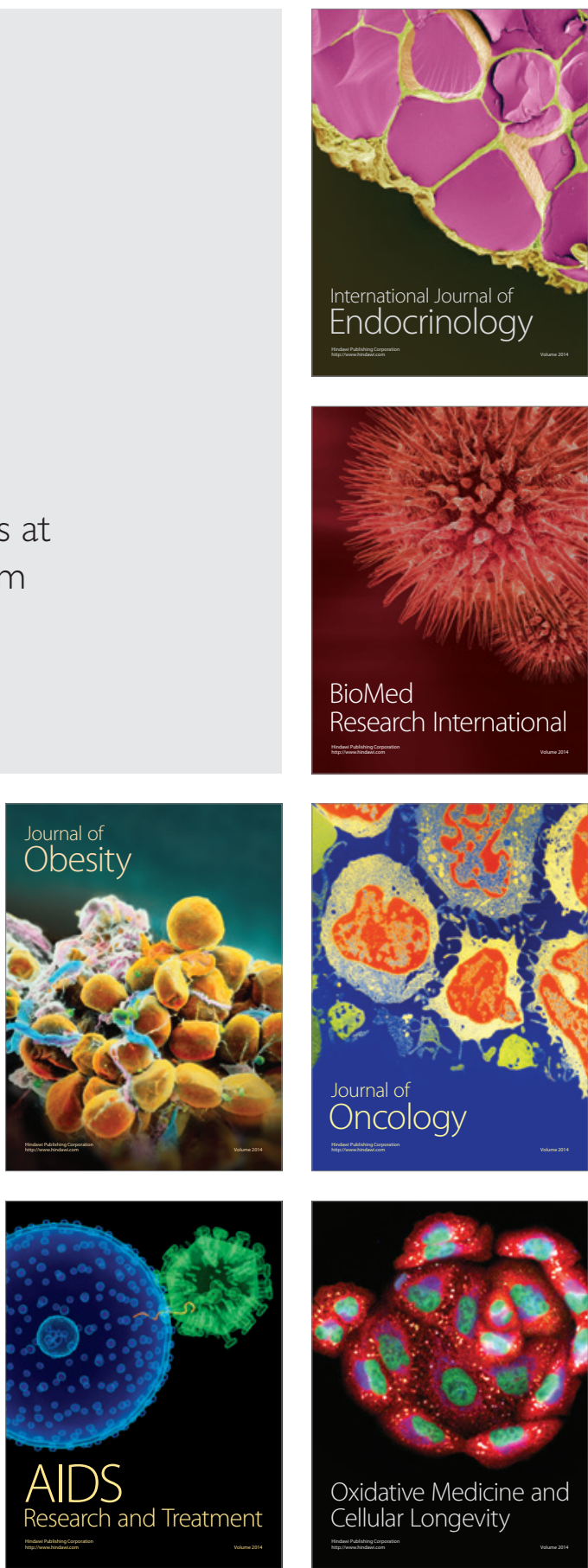